\title{
Major Public Health Problems in Nigeria: A review
}

\author{
Faisal Muhammad ${ }^{1}$, Jamil Hassan Abdulkareem², ABM Alauddin Chowdhury ${ }^{3}$ \\ ${ }^{1}$ Research Assistant; ${ }^{3}$ Assistant Professor; Department of Public Health, Daffodil International University, Dhaka; Bangladesh. \\ ${ }^{2}$ Lecturer, Department of Environmental Health, New Gate College of Health Technology, Minna Niger State, Nigeria.
}

\begin{abstract}
The inadequate programs designed to address the numerous health problems in Nigeria have led to the little improvement in our health status. Besides the continued neglect of the importance of addressing public health issues would make matters worse for poor Nigerians most of who are at the receiving end. This study adopted a qualitative approach, so as to adequately describe the study aims and objectives. The data for this study were collected through scientific database sources, web search engines, direct observation and relevant documents from the Nigerian Ministry of Health. The major public health challenges Nigeria faces are infectious diseases, control of vector some diseases, maternal mortality, infant mortality, poor sanitation and hygiene, disease surveillance, non-communicable diseases and road traffic injuries etcetera. Nigeria is currently working towards achieving the Millennium Development Goals. Despite the collaborative efforts of both Nigerian Government, Donor Agencies and NGOs to provide an efficient and effective health care delivery in Nigeria.
\end{abstract}

Keywords: Public Health, Maternal-Mortality, Malaria, HIV/AIDS, Nigeria.

\section{Introduction}

Nigeria is often referred to as the "Giant of Africa", owing to its large population and economy. ${ }^{1}$ With approximately 182 million inhabitants, Nigeria is the most populous country in Africa and the seventh most populous country in the world. Nigeria has one of the largest populations of youth in the world. ${ }^{2}$ Nigeria is the most populous country in Africa with more than 170 million people. ${ }^{3}$ It is a nation made of more than 250 ethnic groups, 380 languages, and a diverse range of cultural and religious beliefs and practices. Health problems in Nigeria are challenging, but addressing them using public health principles is necessary to support stability in this important area of the world.

The inadequate programs designed to address the numerous health problems in Nigeria have led to the little improvement in our health status. Overall life expectancy at birth is 54 years; infant mortality rate is 86 per 1000 live birth while maternal mortality ratio is 840 per 100,000 live births. ${ }^{4}$ Besides the continued neglect of the importance of addressing public health issues would make matters worse for poor Nigerians most of who are at the receiving end.

Nigerians will continue to die unnecessarily from preventable conditions and disease if there are no proper programs designed to address each of these problems. The first WHO Global Status Report on non-communicable disease listed Nigeria and other developing countries as the worst hit with deaths from non-communicable diseases. ${ }^{5}$ These diseases with a rising burden in Nigeria include cardiovascular disease, cancer, diabetes, chronic respiratory diseases, sickle cell disease, asthma, coronary heart disease, obesity, stroke, hypertension, road traffic injuries and mental disorders. According to the 2011 World Health Statistics, malaria mortality rate for Nigeria

\section{Practice Points}

- Nigeria is often referred to as the "Giant of Africa", owing to its large population and economy, with approximately 182 million inhabitants.

- Communicable and infectious diseases are the major health problem in Nigeria.

- The top causes of death in Nigeria are; malaria, lower respiratory infections, HIV/AIDS, diarrheal diseases, road injuries, protein-energy malnutrition, cancer, meningitis, stroke and tuberculosis.

- Malaria remains the foremost killer disease in Nigeria. It accounts for over $25 \%$ of under 5 mortality, $30 \%$ childhood mortality and $11 \%$ maternal mortality.

- Nigeria still has the second highest HIV burden in the world, About 3.4 million Nigerians are living with HIV.

is 146 per 100,000 population. ${ }^{4}$ Nigeria has one of the highest Tuberculosis burden in the world (311 per 100,000 ) resulting in the largest burden in Africa. ${ }^{6}$ The proper design of programs to address the public health problems in Nigeria will no doubt go a long way in improving the health status of the people. Though there are programs designed to address some of the health issues, there is a need to solve many other health problems.

The Nigerian health sector is currently facing significant challenges which include, among others an uncompleted agenda on the containment of infectious

Correspondence: Faisal Muhammad, Research Assistant, Department of Public Health, Daffodil International University, Dhaka; Bangladesh. Email: fokkanya@yahoo.com. 
disease, as well as the rapid and on-ongoing emergence of non-communicable diseases. Life expectancy at birth in Nigeria stands at 46.8years for men and 48.4years for women, while there is a dire shortage of health workers as a result of economic and social brain drain. ${ }^{7}$ It has been estimated that there are only 27 physicians per 100,000 people in Nigeria. ${ }^{7}$ These multifaceted challenges are compounded by arisen economic policies and socio-political factors in the country history a limited institutional capacity to provide efficient responses at a population level. Lack of commitment on the part of the government, especially during military rule has worsened the fragile sector. ${ }^{8}$ According to the UNDP report 2004 both public and private expenditure as a percentage of GDP is $0.8 \%$ and $2.6 \%$ respectively. Successive governments have on many occasions set out their commitment to this sector, but unfortunately, this rhetoric is not met with tangible and enduring actions, particularly in the area of health system financing. There is a window of opportunity available for initiatives aimed at enhancing the emergence of an integrative approach to public health problems in Nigeria, taking into account the social, cultural and economic determinants of health and also structuring the health system as an efficient channel for health services delivery.

The current national health policy have concise statements on policies of health programs such as HIV/ AIDS, Malaria, Immunization, Population, Reproductive Health, Control of Onchocersiesis, Tuberculosis and Leprosy, Blood Transfusion, Elimination of Female Genital Mutilation, Adolescent health, Food and Nutrition, Child Health, Drug \& Food hygiene and safety. ${ }^{5}$ The major thrusts of the health policy relate to issues such as National Health Systems and Management, National Healthcare Resource, National Health information system, partnerships for Health development, health research and National Health care laws. This was the outcome of stakeholders submissions on what the national health policy should look like. The public's perception of the revised national health policy is unknown. Though there has been some improvement in the health status of Nigerians but the difference is insignificant. For instance, the achievement of the millennium development goals (MDGs) to which Nigeria, like other countries committed to achieve by 2015 remains to be seen. 5,9

The issue of equity, accessibility, affordability, quality, effectiveness and efficiency which are the overall policy objectives of the revised national health policy still persistent. The primary health care on which the Nigerian health care system is based has not helped in effectively solving the numerous health problems in Nigeria. Cost effective interventions for priority public health issues such as non-communicable diseases, injuries, maternal and child health etcetera are hardly being used. Inter-sectoral cooperation and collaboration between the different health-related ministries still remains a major issue. Each day there are about 1,000 new infections of HIV in Nigeria. ${ }^{10}$ The World Health Statistics of 2011 also showed that the number of reported cases of malaria increased from 2,834,174 in 2008 to $4,295,689$ in $2009 .^{4}$ National health systems remain weak while its management is ineffective and inefficient. There is no equitable distribution of human resources between urban and rural area. Over 70 percent of doctors are in urban areas where only 48 percent of the population live, leaving 52 percent of the population who live in the rural area at the mercy of inadequate health personnel. ${ }^{4}$

The National Emergency Response and Preparedness Team was constituted by the Federal Ministry of Health $(\mathrm{FMOH})$, Nigeria in recognition of the importance of disease prevention and control. ${ }^{11}$ But having the department of public health which is responsible for public health, emergency preparedness and response as well as disease prevention and control under the FMOH has not helped matters besides the mission of public health as defined by the Institute of Medicine (IOM) which is "fulfilling society's interest in assuring the conditions in which people can be healthy" has not been achieved by the department of public health under FMOH. Every year, Nigeria records an outbreak of different diseases which kill innocent Nigerians in their thousands. ${ }^{11}$ Despite this yearly occurrence of outbreak, there has not been an effective and efficient emergency response and disease prevention system in Nigeria. That is why the department of public health under the FMOH needs to be upgraded into an agency with or as part of the FMOH that will focus on emergency planning and preparedness; disease prevention and control; and promotion of the health of Nigerian people while the FMOH focus on other issues. ${ }^{12}$

The FMOH is a public health agency and service of government but its response system is slow. The role of the department of public health in disease prevention and control under the FMOH has not been felt over the years. Epidemic prone disease such as Cerebral Spinal Meningitis, Cholera, Measles, Lassa Fever, Yellow Fever as well as other disease of public health importance like diarrhoeal, malaria, plague, tuberculosis, pertussis, onchocerciasis, pneumonia, HIV/AIDS, STI, hepatitis B and so on, require a specialized agency to mitigate their effect on the population. The establishment of a National Public Health Agency (NPHA) will increase the capacity of FMOH to provide prompt and effective public health interventions to Nigerians. ${ }^{11,12}$ This must be done if the government must reduce the incidence of disease and disability. Surveillance, emergency preparedness, disease prevention and control will be the main responsibility of the NPHA thereby relieving the FMOH of the burden of having to ensure the health promotion of Nigerians along with other functions of the Ministry. The formulation of NPHA is imperative if the public health problems in Nigeria must be addressed besides the agency will be concerned with threats to the health of the Nigerian people.

Cholera outbreak in Nigeria estimated as 3 to 5 million cases annually and 100,000 to 150,000 deaths yearly. ${ }^{13}$ It was the worst outbreak in Nigeria in recent years. The number of cases was three times higher than that of 2009 and seven times higher than in 2008. ${ }^{11}$ According to UNICEF, UN \& WHO, women and children account for four out of every five cases. ${ }^{11}$ This study was aimed to identify the major public health problems in Nigeria.

\section{Materials and methods}

The search for this review was conducted throughout the period of the study (January to July 2016) to track new developments and published reports and articles. This review was carried out according to a method 
(York Methodology) outlined by Arksey and O'Malley 2005 from the University of York, United Kingdom. ${ }^{14}$ This study adopted a qualitative approach, so as to adequately describe the study aims and objectives. The study was based on both primary and secondary data. The primary data for this study were collected through scientific database sources and web engine searches, i.e. Public Health Foundation of Nigeria (PHFN), United Nation International Children's Emergency Funds (UNICEF), World Health Organization (WHO), Millennium Development Goals (MDGs), United Nation Program on HIV/AIDS (UNAIDS), United States Agency for International Development (USAID) and relevant articles from African Journals Online. Secondary data were based on direct observation and relevant documents/reports from the Nigerian Ministry of Health (FMOH).

Related articles, reports, and web page documents those that met inclusion criteria for this study were selected and reviewed as well. The inclusion criterion was all the data related to public health issues and challenges in Nigeria (2004 onwards). Reports not wholly focused on the topic of this study were excluded. All the retrieved publications were reviewed with emphasis on the public health issues in Nigeria.

\section{Results and Discussion}

Nigeria faces many public health problems and challenges. ${ }^{15}$ The health issues that Nigeria faces are infectious diseases, sewage disposal, health insurance, water supply, air pollution, noise pollution, environmental radiation, housing, solid waste disposal, disaster management, control of vector some diseases, doctorpopulation ratio, population-bed ratio, population per health facility, payment system/methods, utilization of care, access to care, improper co-ordination of donor funds, maternal mortality, infant mortality, health financing, poor sanitation and hygiene, incessant doctors strike, disease surveillance, smoking of tobacco, brain drain, rapid urbanization, non-communicable diseases, alcohol abuse, environment degradation, road traffic injuries etcetera.

The top 10 causes of death in Nigeria are as follows: ${ }^{3}$

- Malaria (20\%)

- Lower Respiratory Infection (19\%)

- HIV/AIDS (9\%)

- Diarrheal Diseases (5\%)

- Road Injuries (5\%)

- Protein Energy Malnutrition (4\%)

- Cancer (3\%)

- Meningitis (3\%)

- Stroke (3\%)

- Tuberculosis $(2 \%)$

\section{Public health Issues}

Globally about 58 million people died in the year 2005, however more than 10 million (about 18.8 percent) were from the WHO African Region. ${ }^{16}$ More than three-fifths (64 percent) of the death that occurred in the region resulted from HIV/AIDS $(19 \%)$, lower respiratory infections $(10 \%)$, malaria $(8 \%)$, diarrhoeal diseases $(7 \%)$, cerebrovascular disease $(4 \%)$, ischaemic heart disease $(3 \%)$, tuberculosis $(3 \%)$, measles $(3 \%)$, low birth weight $(2 \%)$, birth asphyxia and birth trauma $(2 \%)$ and maternal conditions $(2 \%)$. Nevertheless the effective public health interventions that could have prevented most of deaths exist, but coverage is low due to weak and under-resourced health systems. Some of the weaknesses can be attributed to challenges related to leadership and governance; health workforce; medical products, vaccines and technologies; information; financing; and services delivery.

In Nigeria communicable and infectious diseases are the major health problem. ${ }^{3}$ Nigeria has slowly entered the era of "disease of the affluent" Diseases like Hypertension, Cancer, Obesity etc. Most of the Nigerians (young and old) die of different preventable diseases such as HIV/AIDS, tuberculosis, malaria, vaccine preventable disease of childhood, diarrheal, acute respiratory infections, maternal and neonatal conditions due to inadequate investment in preventive services. ${ }^{3,15}$

A country with a largely unhealthy population cannot progress economically, increase her Gross Domestic Product per capita nor experience economic development which is measured primarily by economic growth. Nigerians young and old die of different preventable diseases such as HIV/AIDS, tuberculosis, malaria, vaccine preventable disease of childhood, diarrheal, acute respiratory infections, maternal and neonatal conditions due to inadequate investment in preventive services. The direct and indirect cost of illness have also continued to pauperize most of the citizens and this affect economic growth as well as development. The sooner governments begin to improve investment on health of the people, the better. African countries like Liberia, Malawi, Burkina Faso, Djibouti, Botswana and Rwanda that are poor than Nigeria have met the target of the Abuja Declaration of 2001 in which all African Health Ministers agreed upon a $15 \%$ budgetary allocation to health. ${ }^{15}$

\section{Malaria}

Malaria remains the foremost killer disease in Nigeria. ${ }^{18}$ It has the highest burden of disease in Nigeria with an estimated 300,000 children dying of malaria each year. It accounts for over $25 \%$ of infant mortality (children under aged one), $30 \%$ of childhood mortality (children under five), and $11 \%$ of maternal mortality. At least $50 \%$ of the population has at least one episode of malaria annually, while children aged less than 5 years have 2 to 4 attacks annually. ${ }^{19}$ Malaria is mostly severe among pregnant women and children less than 5 years of age, due to their relatively lower levels of immunity. To address the importance of both malaria treatment and prevention, the Society for Family Health (SFH) malaria programming centers on Pre-Packaged Therapy (PPT) and Long Lasting Insecticide Treated Nets (LLINs). Recently, the Federal Ministry of Health has implemented the new treatment policy on malaria which includes the use of Artemisinine-based Combination Therapy (ACT) as the new first line drug for the treatment of uncomplicated malaria. ${ }^{20} \mathrm{~A}$ new brand of ACT for Children KidACT was developed and launched in 2008. The brand is heavily subsidized for affordability to poor and 
vulnerable Nigerians who bear the brunt of malaria. Like other NGOs, SFH is promoting the government policy and in addition distributes PermaNet which is a long-lasting insecticide-treated net. ${ }^{19,20}$ The nets are inexpensive and distribution is an easy way of preventing malaria and possibly other vector-borne diseases from burdening the health and economic wellbeing of Nigerians.

\section{HIV/AIDS}

Nigeria has 2.9 million people living with HIV/AIDS, the largest number in the world after India and South Africa. The HIV/AIDS pandemic, which has already left at least 930,000 children orphaned, and the high rates of maternal death and disability, are outstanding public health issues in Nigeria. ${ }^{21} \mathrm{~A}$ high incidence of unsafe abortion is driven by legal restrictions and social stigma, while an extremely low rate of contraceptive use contributes to an estimated 1.4 million unintended pregnancies each year. ${ }^{15}$

In response to the growing HIV/AIDS pandemic treatment, USAID/Nigeria provides antiretroviral drugs and services to eligible patients, as well as laboratory support for the diagnosis and monitoring of HIVpositive patients. The treatment program features reduced target costs, cost-leveraging, and health care worker training by all implementing partners to harmonize and standardize treatment services. This program provides much-needed drugs to many Nigerians with HIV but can nowhere near address the growing HIV/AIDS infection rate. The Nigerian government has set a 2010 goal of providing universal access to HIV prevention, care, and treatment. To this end, it has implemented a number of strategies to scale up HIV services, including a national counseling and testing program and increased collaboration with external donors and non-governmental organizations. $^{21,22}$

\section{Reproductive health and child survival}

Despite considerable gains in the past decade, Nigeria's reproductive health indicators are still very poor. Country-wide, the total fertility rate is 5.7 children per mother, with a contraceptive prevalence rate of less than $10 \% .{ }^{15}$ Furthermore, these statistics mask wide regional variations, for example, the total fertility rate in the northwestern region is as high as 7.3, with a contraceptive prevalence rate of $3 \%{ }^{23}$ Lack of sexual health information and services make young people vulnerable to sexually transmitted infections (STIs) and unintended pregnancy. Nevertheless, Nigerian government both at Federal and State is working in collaboration with many organizations to improve adolescent reproductive and sexual health through advocacy and prevention programming.

Child survival in Nigeria is threatened by nutritional deficiencies and illnesses, particularly malaria, diarrhoeal diseases, acute respiratory infections (ARI), and vaccine preventable diseases (VPD), which account for the majority of morbidity and mortality in childhood. ${ }^{15,24}$ Other threats include high maternal morbidity and mortality. Regarding child health, the country has adopted and implemented to a certain extent a number of major global initiatives affecting children, such as the Safe Motherhood Initiative and its follow-up Making Pregnancy Safer, Baby-Friendly Hospital Initiative
(BFHI), and Integrated Management of Childhood Illness (IMCI). Others are Roll Back Malaria Initiative (RBM), Elimination of Iodine a Dependent Diabetes, Vitamin A Deficiency Control, and National Program on Immunization (NPI), the latter with a special emphasis on the eradication of poliomyelitis. Yet the impact of interventions in child survival to a large extent have not achieved as much as would have been expected despite the amount of funds and resources that have been put into these programs. For instance, Nigeria is still one the three countries of the world harboring and spreading the three serotypes of wild polio viruses to different parts of the world despite ongoing intensive immunization activities because of non-compliancy to polio vaccination. ${ }^{25}$ It may be recalled that in 2003 , there was a political propaganda that Polio vaccine contained infertility agents, spread HIV and was reported generally unsafe. ${ }^{26}$ Although the safety of the polio vaccine was later proven beyond all reasonable doubt globally, and frantic efforts have been made to disabuse the minds of the people, pockets of parents still refuse their wards/children to receive the vaccine.

The crude death rate, life expectancy, infant mortality rate, human development index, maternal mortality rate, the proportion of GNP spent on health services are testaments to this fact, the above indicators do not show that Nigeria is a country that supports the survival of the Nigerian people in a world where their fellow human beings live long enough due to increased life expectancy and proper investment in the health of the people. ${ }^{5}$ It is often believed that only wealthy nations can possibly increase life expectancy and reduce infant and maternal mortality rate as recorded in countries like United Kingdom, Germany, Italy, Spain, France, Japan, Canada, Australia, Sweden, Chile, USA etcetera but donor funds to developing countries which is expected to bridge the financing gap have not been properly utilized for the improvement of the citizen's health status. ${ }^{5}$

\section{Tuberculosis control}

Nigeria is ranked 4th among the 22 worst affected countries in the world and the first in Africa. ${ }^{27}$ As such, about 460,000 new TB cases occur yearly in Nigeria. ${ }^{22}$ Lagos state carries $8.4 \%$ of Nigeria TB burden and consistently has been responsible for about $11 \%$ of the cases of TB registered in Nigeria. The State program is implementing the internationally recommended STOP TB Strategy. ${ }^{22}$

USAID/Nigeria implements its HIV/AIDS and TB activities under a comprehensive approach with other United States Government agencies, including the Centers for Disease Control and Prevention and the Department of Defense, which are all working as part of the President Emergency Plan for AIDS Relief (PEPFAR). ${ }^{4}$ Activities are designed to reduce TB transmission, improve diagnosis, and manage multi-drug-resistant-TB cases, especially among HIV positive patients.

Routine HIV testing is also a priority in USAID TB Directly Observed Treatment Short-Course. The control and prevention of Tuberculosis in contemporary times has many faces and challenges. These, among others, include the impact of HIV/AIDS and the emergence of multi-drug resistant tuberculosis (MDR-TB). The HIV/ 
AIDS pandemic is not only fueling the burden of tuberculosis but also poses great challenge to its diagnosis and management. The recorded HIV prevalence among TB cases in Nigeria is estimated at $27 \%$.

\section{Leprosy control Program}

It has been estimated that about 5,000 leprosy cases occur yearly. ${ }^{22}$ By the 1940 s and 50s Nigeria was ahead of many countries in its leprosy control activities. There has been significant reduction in the registered prevalence of leprosy with some evidence of reduced transmission. This has been attributed to increased and sustained control activities resulting in the elimination of the disease as a public health problem at national level. However, there are still endemic pockets at the sub-national level.

An issue of concern in leprosy control remains the rehabilitation of a large number of ex-leprosy patients who have been cured of leprosy but have disabilities. In collaboration with many NGOs, effective, integrated leprosy control programs in which both female and male patients are identified, diagnosed and treated in the early stages of leprosy by the health system are ongoing. These programs also work to prevent and reduce impairments associated with leprosy, and to provide appropriate rehabilitation and education and vocational training opportunities for persons affected by leprosy. ${ }^{28}$

\section{Conclusion}

Nigeria is currently working towards achieving the Millennium Development Goals. To reach the targets for the reduction of child mortality, which form MDG 4, Nigeria should reduce under-five deaths per 1,000 live births to 71 , and increase measles immunization to 100 per cent by 2015 .

Despite the collaborative efforts of both Nigerian Government, Donor Agencies and NGOs to provide an efficient and effective health care delivery in Nigeria, confronting problems render these efforts much less than desired. Some of these problems or challenges include both emerging and re-emerging health problems such as HIV/AIDS pandemic, inadequate payment of health workers salaries, poor quality of care, inequitable health care services, brain drain, and irrational appointment of health workers among others. A new global burden has revealed that malaria and HIV are still leading cause of death in Nigeria killing more than 190 thousand and 130 thousand people respectively.

\section{Recommendations}

The study put forward following recommendations:

- Government should improve its plan on how to tackle the major public health issues in the country (i.e. HIV/AIDs, Tuberculosis and Malaria remain major health issues in Nigeria) and it also try more on the incidence of drug resistant $\mathrm{TB}$ and extremely drug resistant $\mathrm{TB}$ in order to tackle them.

- Efforts on the vertical transmission of HIV infection should be double to ensure the reduction of mother-to-child transmission.
Government should be committed to the new Global Fund initiative in combating HIV/AIDS, TB and malaria and it has to do everything possible to ensure the reduction of the three identified diseases in line with the Millennium Development Goals (MDGs) targets in 2015.

\section{Acknowledgments}

We thank all the staff of Public Health Department Daffodil International University, Dhaka Bangladesh for their support and advices.

\section{Competing interests}

The authors declare that they have no competing interests.

\section{References}

1. Peter H. Nigeria Giant of Africa. London : Swallow Editions, 1987.

2. The CIA World Fact Book 2014. Skyhorse Publishing, Inc. 2013.

3. Centre for Disease Control and Prevention (CDC), Nigeria report 2013. https:// www.cdc.gov/globalhealth/countries/nigeria/ why/default.htm (accessed 17 June 2016).

4. World Health Organization. World Health Statistics Report-2011. Geneva: WHO 2011.

5. Bolaji SA. Addressing the Public Health Challenges Nigeria Faces. https:// www.inigerian.com/addressing-the-public-health -challenges-nigeria-faces/ (accessed 20 May, 2016)

6. World Health Organization. Global Tuberculosis Report-2009. Geneva: WHO 2009.

7. Public Health Foundation of Nigeria. http:// www.phfn.org/DynamicPage.aspx?pageid $=23$ (accessed 2 June 2016).

8. United Nations Development Programme. Nigerian Human Development Report-2004. New York:UNDP, 2004.

9. United Nations Development Programme. What Will It Take to Achieve the Millennium Development Goals? - An International Assessment. New York: UNDP, 2010.

10. United Nation Programme on HIV and AIDS (UNAIDS). Country Progress Report. Nigeria: UNDP 2010 .

11. Bolaji SA. The national public health agency alternative; National daily newspaper, 2012. http://old.nationaldailyng.com/editorial/ comments-and-issues/4686-the-national-publichealth-agency-alternative (accessed June 2016).

12. Elvis EI, Akinola AF, Ikeoluwapo OA. An overview of disease surveillance and notification system in Nigeria and the roles of clinicians in disease outbreak prevention and control. Niger Med J 2015;56(3):161-8.

13. World Health Organization. Cholera Annual Report 2011: Weekly Epidemiological Record, Volume 87:289-304, Geneva:WHO, 2012. 
14. Arksey H, O’Malley L. Scoping studies: towards a methodological framework. Int $J$ Soc Res Methodol 2005; 8(1):19-32.

15. Baba M, Omotara B. Nigeria Public HealthGains and Challenges. College of Medical Sciences, University of Maiduguri, Nigeria; PEAH 2012. http://www.peah.it/2012/09/ nigerias-public-health-gains-and-challenges/ (accessed June 2017).

16. World Health Organization (WHO). Health Statistics and Information Systems: Global Health Estimates. http://www.who.int/healthinfo/ bod/en/index.html (accessed June 2017).

17. World Health Organization. Strengthening Health Systems to Improve Health Outcomes: WHO's framework for action. Geneva: WHO, 2007.

18. Federal Ministry of Health Nigeria (FMOH) and Population Services International (PSI) 2002. http://www.psi.org/nigeria (accessed June 2017).

19. Nigeria Demographic and Health Survey (NDHS). Demographic and Health Survey Report-2008, Nigeria: NDHS 2009.

20. Society for Family Health Nigeria (SFH). Malaria Prevention and Treatment. http:// www.sfhnigeria.org/? $\mathrm{p}=708$ (accessed June 2017).

21. Planned Parenthood. Nigeria country program 2012. www.plannedparenthood.org/about-us/ newsroom.htm (accessed June 2017).
22. Federal Ministry of Health Nigeria (FMOH) and National Tuberculosis and Leprosy Control Programme (NTBLCP). Workers Manual. Revised $5^{\text {th }}$ Edition. Abuja: Federal Ministry of Health Nigeria, 2010.

23. Pathfinder International Organization. What We Do in Nigeria. http://www.pathfind.org/ countries/nigeria/ (accessed June 2017)

24. Olanrewaju O, Odubunmi AS. Demand for Child Healthcare in Nigeria. Glob J Health Sci 2012 Nov; 4(6): 129-40.

25. United Nations International Children's Emergency Fund (UNICEF). Global Polio Eradication Initiative (GPEI) Status: Endemic https:// www.unicef.org/immunization/polio/files/ UNICEF_GPEI_country profile_NIG_3May201 2.pdf (Accessed June 2017).

26. Guerrera M. Finish Polio: Evolutionary medicine principles and the eradication of polio in evolutionary medicine, Central Connecticut State University. Topics in Biology (Bio 490/ 540), 2007.

27. Dim CC, Dim NR, Morkve O. Tuberculosis: a review of current concepts and control Programme in Nigeria. Niger $J$ Med 2011; 20 (2):200-6.

28. Canadian International Development Agency. Project Browser. Project profile for Leprosy Control project in Sokoto State. Grand Rapids, Michigan: CIDA, 2012. 